\title{
SUBSIMPLE, INJECTIVE, RETRACT
}

\section{by SHALOM FEIGELSTOCK and AARON KLEIN}

(Received 7th November 1983)

Simple and subsimple objects were introduced in [6]. It was shown that if there are enough simple objects in a category $\mathscr{C}$, then there is no room for injectives in $\mathscr{C}$. This idea was exploited in [6] and [2] to show that several classes of groups, rings and classes belonging to other categories do not possess non-trivial injectives or retracts. In this note, the above results will be strengthened by introducing a weaker condition than subsimple of [6]. As a consequence, and by employing some embedding theorems, we show that some important classes do not possess non-trivial retracts.

All the categories are assumed to have a zero object.

Definition. Let $\mathscr{C}$ be a full subcategory of a category $\mathscr{D}$. An object $A$ of $\mathscr{C}$ will be called $\mathscr{D}$-subsimple if there exist $S \in \mathrm{ob} \mathscr{D}, T \in \mathrm{ob} \mathscr{C}$ such that $A$ is a proper subobject of $S$, $S$ is a subobject of $T$, and $S$ is simple in $\mathscr{D}$ [6, Definition (i)].

Obviously a subsimple object in a category $\mathscr{C}$, as defined in [6], is a $\mathscr{C}$-subsimple object.

Theorem 1 of [6] and Lemma 1 of [2] are extended as follows. (Let $\mathscr{C}$ and $\mathscr{D}$ be as in the definition above.)

Theorem 1. If a non-zero $I \in \mathrm{ob} \mathscr{C}$ is an extremal quotient in $\mathscr{D}$ of a $\mathscr{D}$-subsimple object $A \in \mathrm{ob} \mathscr{C}$, then $I$ is not injective in $\mathscr{C}$.

Proof. Assume $I$ is injective in $\mathscr{C}$. Let $A \stackrel{m}{\rightarrow} S \stackrel{h}{\rightarrow} T, m$ non-invertible, $S$ simple in $\mathscr{D}$, $T \in \mathrm{ob} \mathscr{C}$, and let $A \stackrel{\mathscr{E}}{\rightarrow} I$ be extremal [4, 17.9]. As $I$ is injective in $\mathscr{C}$ and $h m \in \mathscr{C}(A, T)$, there exists $f \in \mathscr{C}(T, I)$ such that $f(h m)=e$. Clearly $f h$ is a monomorphism since $f h \neq 0$, as $I \neq 0$. Hence $f h$ is invertible, since $e$ is extremal. So $m$ is an extremal epimorphism and a monomorphism, hence invertible. Contradiction.

CoroHary 2. (i) A full category of groups containing the free groups and the symmetric groups does not possess non-zero injectives. (ii) A full category of $J$-algebras, $J$ an integral domain, containing the free J-algebras and the algebras of endomorphisms of J-modules does not possess non-zero injectives.

Theorem 3. A $\mathscr{D}$-subsimple object $A \in \mathrm{ob} \mathscr{C}$ cannot be a retract in $\mathscr{C}$. ( $\mathscr{C}$ and $\mathscr{D}$ are as in the definition above.) 
Proof. Assume $A$ is a retract in $\mathscr{C}$ and $A \stackrel{m}{\rightarrow} S \stackrel{h}{h} T$ as in the proof of Theorem 1. Since $h m \in \mathscr{C}(A, T)$, there exists $g \in \mathscr{C}(T, A)$ such that $g(h m)=1$. But $g h \neq 0$ since $A \neq 0$, so $g h$ is a monomorphism, hence invertible. So $m$ is invertible. Contradiction.

Theorem 4. There are no non-trivial retracts in:

(i) the class of finitely generated groups, $\mathscr{F} g \mathscr{G} r$; (ii) the class of $n$-generator groups, $n$ a positive integer, $n \mathscr{G} r$; (iii) the class of countable, locally finite groups, $\mathscr{C} \mathscr{L} f \mathscr{G} r$; (iv) the class of finitely generated groups with solvable word problem.

Proof. (i): Let $G$ be a finitely generated group. In particular $G$ is countable, so by a theorem of Boone and Higman [1], there exists a simple countable group $H$ such that $\mathrm{G} \varsubsetneqq H$. Hence, by a theorem of Higman, B. H. Neumann and $H$. Neumann [5, Theorem 4], there exists a 2-generator group $K$ with $H \leqq K$. It follows that $G$ is $\mathscr{G}_{r}$-subsimple in $\mathscr{F} \mathrm{g} \mathscr{G}$ r, so by Theorem 3 , there are no non-trivial retracts in $\mathscr{F} g \mathscr{G}$.

(ii): The case $n=1$ can be easily proved directly. Let $n>1$. Again by the theorems of [1] and [5] mentioned above, every group in $n \mathscr{G} r$ is $\mathscr{G} r$-subsimple in $n \mathscr{G} r$, hence there are no non-trivial retracts in $n \mathscr{G} r$, Theorem 3.

(iii): Let $G$ be a countable, locally finite group. By a theorem of P. Hall [3], there exists a simple, countable, locally finite group $H$ such that $G \supsetneqq H$. Put $K=H$, and apply Theorem 3 to obtain that there are no non-trivial retracts in $\mathscr{C} \mathscr{L} f \mathscr{G}$.

(iv): Same proof as for (iii) but instread of P. Hall's theorem we employ a theorem of Thompson [7] to embed any finitely-generated group with solvable word problem into a simple group of the same sort.

\section{REFERENCES}

1. W. W. Boone and G. Higman, An algebraic characterization of groups with soluble word problem, J. Austr. Math. Soc. 18 (1974), 41-53.

2. S. Feigelstock and A. Klein, Retracts and injectives, Canad. Math. Bull. 25 (4) (1982), 462467.

3. P. Hall, Some constructions for locally finite groups, J. London Math. Soc. 34 (1959), 305319.

4. P. Herrlich and G. E. Strecker, Category Theory (Allyn and Bacon, Boston, 1973).

5. G. Higman, B. H. Neumann and H. Neumann, Embedding theorems for groups, J. London Math. Soc. 24 (1949), 247-254.

6. A. KLeiN, Injectives and simple objects, J. Pure and Appl. Alg. 15 (1979), 243-245.

7. R. J. Tномpson, Embeddings into finitely generated simple groups which preserve the word problem, Word Problem II (Studies in Logic and the Foundations of Math., North-Holland, Amsterdam-New York, 1980), 401-441.

Bar-ILAN UNIVERSITY

RAMAT-GAN IsRAel 52100 